Technical Note

\title{
On the Use of Bright Scatterers for Monitoring Doppler, Dual-Polarization Weather Radars
}

\author{
Marco Gabella \\ MeteoSwiss, via ai Monti 146, CH-6605 Locarno-Monti, Switzerland; marco.gabella@meteoswiss.ch; \\ Tel.: +41-58-460-9692
}

Received: 1 May 2018; Accepted: 21 June 2018; Published: 25 June 2018

Abstract: "Bright" scatterers are useful for monitoring modern weather radars. In order to be "bright", a point target with deterministic backscattering properties should be present at a near range and be hit by the antenna beam axis. In this note, a statistical characterization of the echoes from a metallic tower located on Cimetta, at an $18 \mathrm{~km}$ range and at the same altitude as the Monte Lema radar, is presented. The analysis is based on five clear sky days (1440 samples with a spatial resolution of $1^{\circ} \times 1^{\circ} \times 83.33 \mathrm{~m}$ ). The spectral and polarimetric signatures are striking: The spectrum width is perfectly stable; the mean radial velocity is very stable; the radar reflectivity is also quite stable, with the vertical $(\mathrm{V})$ polarization being more variable than the horizontal $(\mathrm{H})$ one. As far as the polarimetric information is concerned, the daily average of the differential reflectivity is approximately $1 \pm 0.9 \mathrm{~dB}$. The copolar correlation coefficient between $\mathrm{H}$ and $\mathrm{V}$ is remarkably large $(0.9962$, on average) and stable. It is believed that these unique and stable ground clutter signals could be used to monitor operational dual-polarization weather radars.

Keywords: monitoring; quality checks; relative calibration; dual-polarization weather radar

\section{Introduction}

Ground echo is a challenge for operational weather radars, especially as far as hydrological applications and precipitation estimates are concerned. The identification and elimination of ground clutter is a prerequisite for the use of weather radar. Since European radars are often used for the surveillance of complex-orography regions, they are installed out of necessity on the top of a hill or a mountain, where the rejection of clutter is of vital importance, since a clear view from a high site implies a large number of ground clutter pixels. Ground clutter is automatically detected in the operational radar processing chain of meteorological services using certain specific and well-consolidated algorithms and then rejected so that only the radar echoes originating from the weather are retained. In this context, the use of high range resolution (for example, $c \tau / 2<100 \mathrm{~m}$, where $\tau$ is the pulse length), Doppler velocity and dual-polarization information maximizes the probability of having at least some clutter-free radar bins for each Cartesian pixel of the resampled operational product. The distribution of ground clutter echoes can often be fitted using one of the following distributions: Rayleigh (independent randomly distributed scatterers, e.g., [1]), lognormal (e.g., [2]) and Weibull (e.g., [3]). It has been found that, for these skewed-to-the-right distributions, the Log-transformed ratio between the 90th percentile and the algebraic average of the radar reflectivity value generally ranges between 3 and $4 \mathrm{~dB}$ for most ground clutter radar bin cases. Furthermore, in the case of randomly distributed scatterers, the Log-transformed ratio between the algebraic average and the median is $1.6 \mathrm{~dB}$.

Ground-clutter characteristics, as well as their modeling and simulation have been studied extensively, primarily to improve the radar detection of meteorological targets (e.g., [4]). Thanks to the increase in knowledge, together with the exponential increase in computational power for 
modeling and statistical analysis, a novel point of view regarding ground clutter is emerging; it is no longer considered just a disturbance that needs to be rejected and its spatio-temporal characteristics are instead being investigated so that they can be used to monitor the quality of radar hardware. Silberstein et al. [5], for instance, proposed a statistical method, based on the probability distribution of a clutter area close to a ground-based radar, to provide near-real-time estimates of the relative calibration of reflectivity data. The original application was to an S-band radar in a tropical oceanic location; later, Bertoldo et al. [6] successfully applied a similar approach to an X-band radar in the more complex terrain of the Western Alps. The study by Wolff et al. [7] has shown that the technique is capable of monitoring the reflectivity calibration of other S-band radars, given the proper generation of an areal clutter map. The operational implementation of such techniques obviously takes into account the reflectivity values of all the ground clutter low range resolution radar bins (generally, $1^{\circ} \times 500 \mathrm{~m}$ ) that are above a given high reflectivity threshold (e.g., $55 \mathrm{dBZ}$ ) for more than a given percentage of time. It does not consider any other important spectral or polarimetric information that characterizes the ground clutter bins, or perform a temporal analysis of any individual radar bins, even though these could provide useful information for the monitoring of the quality of modern dual-polarization radars. A guiding principle for a comprehensive radar monitoring is in fact to combine as many independent sources of information as possible. These include: Test signals inserted at various points of the radar chains by laboratory test equipment during preventive maintenance; signals transmitted by the radar and measured by an external receiver to check the amplitude and shape of the transmitted radar pulse (e.g., [8]), signals from the Sun during operational weather scanning for the daily monitoring of the pointing accuracy of the antenna [9] and of the relative calibration of the receiver [10-12]; signals from the Sun with maximized Signal-to-Noise ratios (offline Sun-tracking on demand) for the absolute calibration of the receiver [13]; ground clutter maps in clear sky conditions (for instance, percentile maps).

In this technical note, we propose complementing the above-mentioned multi-sensor and multi-signal monitoring approach with a wealth of spectral and polarimetric information associated with an individual scatterer that we have defined as a "Bright Scatterer" (BS). In order to be "bright", a dominant point target, with deterministic backscattering properties, should be present in the radar bin, which has to be located close to the radar and hit by the antenna beam axis. The particular Doppler and polarimetric signatures of the BS emerge in one single, high-range-resolution radar bin: In the case of the Swiss radar, the sampling volume, which depends on the antenna Half Power Beam Width (HPBW) and the pulse length, is $1^{\circ} \times 1^{\circ} \times 83.33 \mathrm{~m}$. Hence, we present detailed information regarding the bin that contains the BS, which is a high metallic tower that has a huge radar cross section (RCS). It is located on Cimetta at an altitude of $1650 \mathrm{~m}$ and has an $18 \mathrm{~km}$ range: the corresponding (HPBW) azimuthal (and elevation) angular resolution is $314 \mathrm{~m}$. The idea of using return signals from a radio tower is not new. In Reference [14], for instance, the focus was on the stability of the radar reflectivity, while in Reference [15] it was on the differential reflectivity. In this note, several other features are analyzed in detail: the mean radial velocity and the spectrum width which complement radar reflectivity. As far as polarimetric information is concerned, in addition to differential reflectivity, the co-polar correlation coefficient and the differential phase shift are presented. A geographic description of the BS location and the radar site is given in Section 2, together with detailed information regarding the radar hardware and scan program. Section 3 presents and describes the results, which are based on the analysis of five clear sky days (1440 samples acquired with a 5-min sampling time): It is shown that the spectral and polarimetric signatures of the BS are impressive, in terms of stability and reproducibility. The findings and their implications are discussed in Section 4, which also presents future research directions. The conclusions are presented in Section 5. 


\section{Materials and Methods}

\subsection{The Dual-Polarization, Doppler Radar Located on Monte Lema at an Altitude of $1625 \mathrm{~m}$}

In 2011, MeteoSwiss started the renewal of its weather radar network, which now consists of five identical, dual-polarization, Doppler, C-band $(5.5 \mathrm{GHz})$ radars [16]. One of them is the Monte Lema radar, which is located at an altitude of $1625 \mathrm{~m} 10 \mathrm{~km}$ northwest of Lugano, very close to the Swiss-Italian border. This is the radar that is dealt with in this study.

Radar hardware monitoring is run continuously: many parameters are monitored and submitted from each radar site to the central server after completion of every single sweep, that is, 20 times in $5 \mathrm{~min}$. These parameters are automatically checked for anomalies and archived for diagnostic analyses. Daily monitoring of the antenna pointing accuracy [9] and of the relative calibration of the receiver has been implemented [11], according to the Dutch/Finnish operational monitoring approach [10,12].

In order to guarantee system stability and reproducibility, instrumental calibration of the radar receiver should be implemented using internal microwave equipment. This is performed at MeteoSwiss using a noise source signal [17], which is inserted every $2.5 \mathrm{~min}$ in both polarization channels of the receiver path, both before the $\mathrm{T} / \mathrm{R}$ limiter and at the entrance of the low noise amplifiers.

The MeteoSwiss state-of-the-art C-band radars determine the standard dual-polarization meteorological quantities (differential reflectivity, $Z^{d r}$, see Section 2.2.4; copolar correlation coefficient, $\rho^{H V}$, see Section 2.2.5; differential phase shift, $\psi_{\mathrm{dp}}$, see Section 2.2.6) from simultaneous horizontal $(\mathrm{H})$ and vertical $(\mathrm{V})$ transmissions. The return signals are measured simultaneously in two parallel $\mathrm{H}$ and $\mathrm{V}$ receiving channels. The advantage of simultaneously transmitting and receiving both polarizations is that $Z^{d r}, \rho^{H V}$, and $\psi_{\mathrm{dp}}$ are determined directly from the same transmitted pulse (which is at zero lag), and are thus not contaminated by Doppler effects that can increase their variance, as is the case when alternating $\mathrm{H}$ and $\mathrm{V}$ polarizations are transmitted. The approach has the additional advantage of not needing a high-power polarization switch. According to measurements performed at the Site Acceptance Tests (i.e., twisting the transmitter or receiver horns of the Radar Target Simulator to the following four configurations: $0^{\circ}, 90^{\circ}$ and $\pm 45^{\circ}$ ), it was possible to verify that the Lema radar is quasi-linear and has a slant of $+45^{\circ}$.

\subsubsection{The Scan Program with Reference to the Target under Investigation}

Each MeteoSwiss radar performs plan position indicator scans at 20 elevations, that is, from $-0.2^{\circ}$ to $40^{\circ}$, over a period of $5 \mathrm{~min}$. Figure 2 in Reference [16] (p. 45) shows the Swiss scan strategy: It starts with sweep \#1 at an elevation of $6.5^{\circ}$ and ends with sweep \#20 at $9.5^{\circ}$ (the first two numbers in the figure). The 3rd and 4th numbers represent the number of antenna rotations per minute and the pulse repetition frequency, respectively, which are relevant for the $1^{\circ}$ angular bin that contains the BS at the lowest angle of elevation. Since the PRF at this angle of elevation is $600 \mathrm{~Hz}$, while the antenna takes $20 \mathrm{~s}$ to compute a $360^{\circ}$ rotation, the BS is illuminated by a rotating antenna for $1 / 18 \mathrm{~s}$. This means that each high-range resolution BS "echo" (observed every $5 \mathrm{~min}$ and referring to the same $1^{\circ}$ azimuthal index) is the result of the spectral analysis of $600 / 18 \cong 33$ pulses, when azimuthal index 349 is used.

\subsection{Physical Radar Observables (and Their Quantization) Coded in High Range Resolution ( $83.33 \mathrm{~m}$ ) Radar Bins}

As previously stated, the $1^{\circ}$ radar bin (azimuth-range cell for a given angle of elevation) has a high resolution range $(83.33 \mathrm{~m})$; its spectral and polarimetric characteristics are quantized using 1 byte, except for the differential phase shift, which is quantized with 2 bytes (namely 65,536 values). Hence, the Digital Number (DN) can vary in binary format from 0 to $255(65,535$ in the case of the differential phase shift). In the case of the MeteoSwiss high range resolution data, $\mathrm{DN}=0$ has no quantitative meaning and instead has a qualitative meaning. In fact, if the Log-transformed ratio between the Signal plus the (unknown) Noise and the (estimated) Noise is smaller than $4 \mathrm{~dB}$, then the echo is considered to be below the Minimum Detectable Signal (MDS). The corresponding radar bin is 
assumed to be WITHOUT (detectable) precipitation, and its DN is set to 0 . More details regarding the physical meaning of the moments used in this study (as well as their quantization) can be found in the following six Subsections.

\subsubsection{Horizontal (H) and Vertical (V) Radar Reflectivity (0th Doppler Moment)}

As mentioned in Section 2.2, a high-range-resolution reflectivity value is coded as long as

$$
10 \log \left(\frac{S+N_{u}}{N_{e}}\right) \geq 4 \mathrm{~dB}
$$

where the numerator represents the power $\left(\mathrm{I}^{2}+\mathrm{Q}^{2}\right)$ of the Signal plus the (unknown) Noise of the current bin, and the denominator is an estimate (performed every $2.5 \mathrm{~min}$ ) of the Noise performed at high Elevation angles $\left(40^{\circ}\right.$ and $35^{\circ}$, see Figure 2 in Reference [16]). In other words, the measured power (Signal plus the unknown Noise) will be at least 2.512 times the estimated Noise that will be recorded as a quantitative value. If not, the radar bin is assumed to be without any (detectable) precipitation and DN is set to 0 (flag value). Let us suppose that the estimate of the Noise is close to the actually measured value: this means that the net Signal should be at least 1.512 times the Noise. As far as quantization is concerned, a value of $0.5 \mathrm{dBZ}$ has been chosen by MeteoSwiss. The linear conversion from $\mathrm{DN}$ to Log-transformed radar reflectivity is the following

$$
Z_{d B Z}=(\mathrm{DN}-64) / 2,
$$

where the radar reflectivity units, $Z$, are $[Z]=\mathrm{mm}^{6} / \mathrm{m}^{3}$, while $Z_{1}\left(Z_{1}=1 \mathrm{~mm}^{6} / \mathrm{m}^{3}\right)$ is the normalization factor necessary to Log-transform $Z$ into the so-called dBZ unit, which, in formula form, is:

$$
Z_{d B Z}=10 \log \left(\frac{Z}{Z_{1}}\right)
$$

In practice, the maximum recorded value for the Monte Lema radar very rarely exceeds $85 \mathrm{dBZ}$ $(\mathrm{DN}=234)$, while $\mathrm{DN}=14(-25 \mathrm{dBZ})$ can, in principle, only be observed within a range of $3 \mathrm{~km}$ or closer (already at the edge of the T-R limiter effects).

\subsubsection{Mean Radial Velocity (1st Doppler Moment)}

Since the radar wavelength is $55 \mathrm{~mm}$ and the PRF of the lowest elevation is $600 \mathrm{~Hz}$, the unambiguous Doppler velocity ( \pm Nyquist) is $\pm 8.25 \mathrm{~m} / \mathrm{s}$. As previously stated, only 255 quantitative values are used in the quantization process: this corresponds to a quantization of $0.061 \mathrm{~m} / \mathrm{s}$. The conversion from DN to the mean radial velocity, VEL, is

$$
\mathrm{VEL}=(\mathrm{DN}-128) / 127 \cdot \text { Nyquist; }
$$

Hence, $\mathrm{DN}=1(255)$ corresponds to a mean radial velocity of $-8.25(+8.25) \mathrm{m} / \mathrm{s}(\mathrm{DN}=0$ is not used). It is worth noting that the minus sign in the MeteoSwiss radar network corresponds to an approaching target (although an approaching target is generally associated with a positive velocity).

\subsubsection{Doppler Spectrum Width (2nd Doppler Moment)}

As far as the spectrum width is concerned, the quantization is also $0.061 \mathrm{~m} / \mathrm{s}$

\subsubsection{Differential (Horizontal/Vertical) Radar Reflectivity}

A first polarimetric observable that can be measured by the MeteoSwiss dual-polarization radar is the differential radar reflectivity, $Z^{d r}$. This is the Log-transformed ratio between the copolar reflectivity 
measured at the horizontal, $\mathrm{ZH}$, and vertical, $\mathrm{ZV}$, polarizations; hence, it is expressed in $\mathrm{dB}$, and a $\mathrm{Z}^{d r}$ of $0 \mathrm{~dB}$ means that $Z H=Z V$. In formula form:

$$
Z^{d r}=10 \log \left(\frac{Z H}{Z V}\right)
$$

This ratio was introduced by Seliga and Bringi [18] to obtain a better estimate of rainfall, since it contributes to reducing the uncertainty associated with rain drop size distributions. In fact, the additional information associated with $Z^{d r}$ is remarkable; however, a proper calibration is crucial for successful quantitative applications. Illingworth [19], for instance, showed that $Z^{d r}$ should be estimated within $0.2 \mathrm{~dB}$ to obtain improved rainfall estimates based on $Z$ and $Z^{d r}$. It is possible to use the so-called birdbath scan (angle of elevation of $90^{\circ}$ ) on rainy days when the altitude of the $0^{\circ}$ isotherm is high enough to be outside the range that is affected negatively by the T-R limiter recovery time to monitor the differential reflectivity backscattered by liquid drops ( $Z^{d r}$ should be $0 \mathrm{~dB}$ ). Another (more uncertain approach) is to derive the mean differential reflectivity value of light rain echoes (for example, $20 \leq \mathrm{ZH} \leq 22 \mathrm{dBZ}$ ) well below the bright band (which should be around $0.3 \mathrm{~dB}$ ).

Offline Sun observations acquired while tracking the Sun $[20,21]$ are more accurate, but only the receiving chain is monitored, and nothing is therefore known regarding the transmitting path. It is shown in Table 3 (4) in Reference [13] that the standard deviation of six (seven) Sun tracking observations (performed between 2 November 2013 and 21 March 2016) for the MeteoSwiss Monte Lema (Albis) radar is $0.08(0.04) \mathrm{dB}$. An interesting and valid alternative to offline Sun-tracking is the automatic daily "Sun-check" [10]: this is an operational monitoring technique that is based on the analysis of solar signals in the polar volume radar reflectivity data produced during the operational weather scan program. It has the advantage of the weather radar scan program not being interrupted; the disadvantage is that the antenna beam axis very rarely hits the center of the Sun: consequently, the Sun-to-Noise ratio of each hit can be significantly worse than for Sun tracking. This is partly compensated for by the fact that only one estimate out of many daily hits is retrieved. In Reference [11] (Table 4), the standard deviation of the daily differential reflectivity during an active solar period in 2014 was $0.06 \mathrm{~dB}$ for Monte Lema (220 days) and $0.05 \mathrm{~dB}$ for the Albis radar (204 days); the dispersion of the difference between $\mathrm{H}$ and $\mathrm{V}$ during the same set of 100 days in 2014 was $0.06 \mathrm{~dB}$ for Albis and $0.08 \mathrm{~dB}$ for the Finish ANJ radar (see Table 3 in Reference [22]). In a recent manuscript that reviews several aspects of the monitoring of dual-polarization receiver using solar hits found in operational scans [12], a dispersion as low as $0.02 \mathrm{~dB}$ was found for ANJ. These figures refer to a shorter period, namely April 2015. If the antenna is not in an environment with small changes in Temperature, then differential reflectivity can easily vary by a few tens of dB [23]: in Figures 4 and 5, page 1890, in Reference [23], it is shown that a $23 \mathrm{~K}$ change in temperature (lasting $8 \mathrm{~h}$ ) occurred at the S-band Firestone site (on 15 January 2015) and a $0.2 \mathrm{~dB}$ drift in $Z^{d r}$ was observed.

Regarding the quantization process, it is worth noting that differential reflectivity is only defined on condition the inequality in (1) is satisfied for both the $\mathrm{H}$ and $\mathrm{V}$ polarizations. If not, $\mathrm{DN}$ is set to 0 . The number of radar bins that contain a valid value of differential reflectivity is obviously smaller than for the $\mathrm{H}$ or $\mathrm{V}$ reflectivity (however, it could be at least the same). In the quantization process based on 255 values the resolution is $15.751 / 255 \cong 0.062 \mathrm{~dB}$.

All the values outside the above-defined range of $15.751 \mathrm{~dB}$ are left- or right-saturated. Since $Z V_{d B Z}$ is also saved in the MeteoSwiss archive (in addition to $Z^{d r}$ and $Z_{d B Z}$ ), the user can retrieve the original, non-saturated value by simply subtracting $Z V_{d B Z}$ from $Z H_{d B Z}$; obviously, in this case, the derived value only has a resolution of $0.5 \mathrm{~dB}$, as can be seen from Equation (2).

\subsubsection{The Copolar Correlation Coefficient}

Another quantity measured by the dual-polarization radar is the correlation between the copolar horizontal, $H H$, and the vertical, $V V$, returns, which is called the copolar correlation coefficient (often referred to as $\rho^{H V}$ or $\rho^{c o}$ ). However, describing the rather complicated nature of this variable 
is beyond the scope of this note. On the one hand, any reader that is interested may refer to the electronic supplement (e06.1) that accompanies the book by Fabry [24]; on the other hand, the detailed treatment of the physics and use of polarimetric quantities has been the subject of entire books, e.g., Bringi e Chandrasekar [25]. The copolar correlation coefficient is the module of the complex correlation coefficient between two orthogonal components (represented by two complex numbers) of the backscattered electromagnetic field within the radar sampling volume: hence, it ranges from 0 (no correlation between the two polarizations) to 1 (perfect correlation). In general, if the sampling volume contains a significant number of ground targets, then, in most cases, $\rho^{H V}$ ranges from 0.75 to 0.95 , for example. However, for the bright scatterer in Cimetta that will deal with in the Section 3 , the median of the five daily values is as large as 0.9972 . A maximum value of 0.9998 has been observed.

Regarding the 1-byte quantization, a considerable resolution is attributed (through a logarithmic transformation) to values of $\rho^{H V}$ close to 1 ; hence, low values of $\rho^{H V}$ are compressed into a few Digital Numbers. The corresponding conversion formula from $\mathrm{DN}$ to $\rho^{H V}$ is:

$$
\rho^{\mathrm{HV}}=1.003-\left(10^{-\frac{D N-1}{100}}\right)
$$

$\mathrm{DN}=0$ corresponds to $\rho^{H V}=0$, while $\mathrm{DN}=255$ is obviously not possible.

\subsubsection{The Differential Phase Shift between the Two Orthogonal Polarizations}

A third polarimetric quantity measured by the MeteoSwiss radars is the differential phase shift, $\Psi_{\mathrm{dp}}$, between the phase of the copolar signal at the horizontal and vertical polarizations, respectively. This difference between the two orthogonal polarization phases arises from two sources:

- a difference in the delay introduced by the scattering of the transmitted wave, which is known as the backscattering phase delay, $\delta_{\mathrm{co}}$;

- a difference in the forward propagation velocity of the two polarizations, which is known as the differential propagation phase, $\Phi_{\mathrm{dp}}$.

In formula form, the differential phase shift, $\Psi_{\mathrm{dp}}$, at any given range, is:

$$
\Psi_{\mathrm{dp}}=\delta_{\mathrm{co}}+\Phi_{\mathrm{dp}}+\Psi_{0}
$$

which is the sum of the backscattering phase delay of the targets at that range; the two-way differential propagation phase that occurred when propagating from the radar to the observed target and then back to the radar; an offset value $\Psi_{0}$, which is the phase difference between the transmitted and received vertically and horizontally polarized waves at the zero range. This constant value depends on the hardware components and design of the radar; at MeteoSwiss, this value was set close to $0^{\circ}$ through a post-facto software adjustment (and it is monitored to ensure it remains constant).

As stated in Section 2.2, the differential phase shift is the only radar observable that is quantized using two bytes. The $\mathrm{DN}$ in binary form ranges from 1 to 65,$535 ; \mathrm{DN}=0$ is not used. The conversion formula from $\mathrm{DN}$ to the value of $\Psi_{\mathrm{dp}}$, which is expressed in degrees, is:

$$
\Psi_{\mathrm{dp}}=(\mathrm{DN}-32768) / 32767 \times 180^{\circ} .
$$

\subsection{The Metallic Tower on Cimetta at an Altitude of $1633 \mathrm{~m}$ : A Particularly Bright Target!}

As stated in the introduction, at the Cimetta range $(\sim 18.14 \mathrm{~km}$, at the center of the radar bin), the radar resolution cell is $83.33 \mathrm{~mm}$ along-range and $314 \mathrm{~m}$ across-range (elevation and azimuth). The picture in Figure 1 shows the mountainous backscattering environment that characterizes the radar bin analyzed in this study. The view is from East to West (an azimuth of $\sim 260^{\circ}$ ) so that it is practically orthogonal to the direction of the incident electromagnetic radar ray (the Azimuth of the radar bin at hand is $349^{\circ}$ ), which, consequently, comes from the left side of the picture. The trees on 
mountain were chopped down centuries ago, so that the land is basically used for grazing, with just a few sparse trees remaining. There are some small summer cottages inside the bin, plus a (larger) hut that is located at $30 \mathrm{~m}$ from the tower (East direction). The tall metallic tower is by far the largest scatterer in the radar bin; it is orthogonal to the incident electromagnetic energy (angle of Elevation of $-0.2^{\circ}$ ). It is as large as $12 \times 12 \mathrm{~m}$ at the base and is covered by solar panels (not visible in the picture, since they are behind the hut). There are all sorts of telecommunication and broadcasting antennas at heights of between 6 and 12m, and then the tower becomes more slender and reaches a height of $90 \mathrm{~m}$ above the ground ( $\sim 1633 \mathrm{~m}$ altitude). This point target exhibits a very large radar cross section and acts as a dominant target in a background of randomly distributed scatterers.

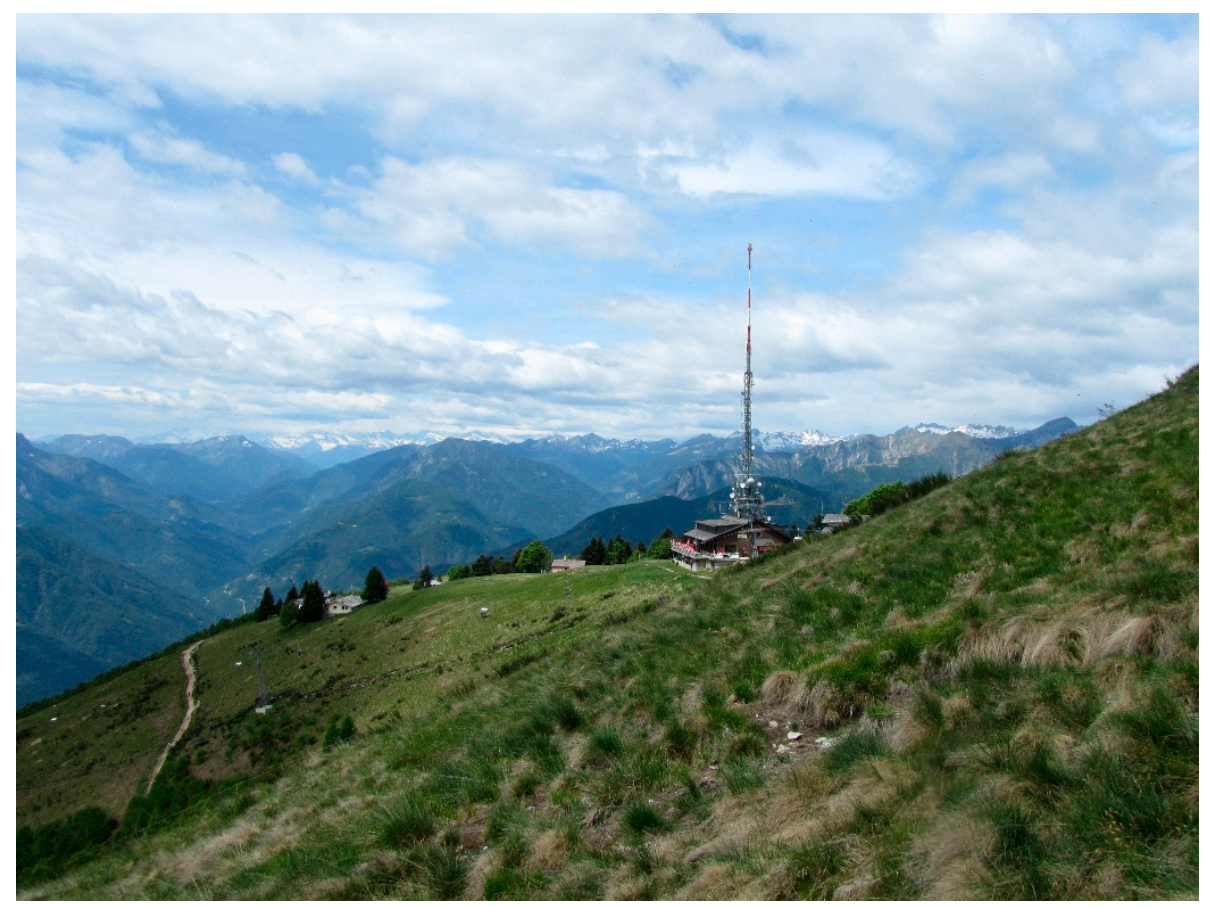

Figure 1. Morphology of the mountainous Cimetta backscattering radar bin: small cottages, the hut, the chairlift and the $90 \mathrm{~m}$ tall metallic tower.

\section{Results}

The present section is aimed at providing a concise description of the experimental results and their interpretation. It is divided into six subheadings that correspond to the six radar observables introduced in Section 2.2, that is, the 2nd and the 1st Doppler moments of the horizontal polarization, backscattered power of both orthogonal polarizations, followed by three polarimetric quantities (differential reflectivity, copolar correlation coefficient and differential phase shift). As stated in the introduction, when the 5-min power returns are analyzed, a random scattering pattern can be expected that is characterized by rapid short-term fluctuations; the phase (2nd and the 1st Doppler moments) is expected to be quite stable because there are rarely moving objects inside the $314 \times 314 \times 83.33 \mathrm{~m}$ radar cells during both calm and windy days. As stated at the end of the introductory section, the results are based on the analysis of five clear sky days; since the sampling is performed every 5 min, there are 1440 samples per day. In turn, each $1^{\circ}$ sample has been derived by the radar signal processor using 33 pulses.

\subsection{Perfect Stability of the 2nd Doppler Moment (Spectrum Width)}

In the absence of atmosphere (no wind, refraction or turbulence) and for a non-rotating antenna, a null spectrum width and radial velocity could be expected; hence, one Digital Number (DN) would 
be sufficient to describe both the 2nd and the 1st Doppler moments. Indeed, no variability at all is found in the spectrum width over the 5 clear sky days of the training data set or over the eleven clear sky days of the evaluation data set (see Section 4). All the $(288 \times 16)$ samples have the same Digital Number $(\mathrm{DN}=1)$. However, this Digital Number does not correspond to the correct interval expected from theory that is $[0.12-0.18] \mathrm{m} / \mathrm{s}(\mathrm{DN}=2)$. This inconsistency will be further investigated together with the radar manufacturer. All considered, any DN value between 1 and 3 could probably be acceptable for meteorological applications $( \pm 0.06 \mathrm{~m} / \mathrm{s})$.

\subsection{On the Very Stable Characteristic of the 1st Doppler Moment (Mean Radial Velocity)}

Despite the presence of a rotating antenna (constant radial velocity of $18^{\circ} / \mathrm{s}$ ), the mean radial velocity is stable; however, it spans two DNs and not only one. The relative frequency of these DNs is shown in Table 1. Equation (4) in Section 2.2 can be used to convert the two DNs into the mean radial velocity. In two (out of three) days of the 2015 year, $\mathrm{DN}=128$ (zero velocity) occurred more frequently than $\mathrm{DN}=127$. Later on, $\mathrm{DN}=127$ is more frequent than $\mathrm{DN}=128$. However, any value between 127 and 129 is probably acceptable for meteorological applications $( \pm 0.06 \mathrm{~m} / \mathrm{s})$.

Table 1. Daily number of occurrences (temporal resolution is $5 \mathrm{~min}$ ) of the mean radial velocity values for the high resolution Monte Lema radar cell on Cimetta that hosts the tall tower.

\begin{tabular}{|c|c|c|}
\hline Date & $\begin{array}{c}\mathrm{DN}=128 \\
\text { Mean Rad. Vel. }=0 \mathrm{~m} / \mathrm{s}\end{array}$ & $\begin{array}{c}\mathrm{DN}=127 \\
\text { Mean Approaching Vel. }=0.06 \mathrm{~m} / \mathrm{s}\end{array}$ \\
\hline 5 January 2015 & 249 & 39 \\
\hline 9 February 2015 & 2 & 286 \\
\hline 10 February 2015 & 286 & 2 \\
\hline 13 October 2016 & 35 & 253 \\
\hline 18 January 2017 & 6 & 282 \\
\hline
\end{tabular}

\subsection{On the Quite Stable Characteristics of the Oth Doppler Moment (Horizontal and Vertical Polarization)}

In the case of a single transmitted signal, backscattered by a mountainous surface of uniform electromagnetic properties (ensemble of independent, randomly distributed scatterers), the incoming signal to the receiver may be assumed to be Rayleigh distributed (i.e., the received power is distributed exponentially, in a similar way to that caused by rain drops, thermal noise, ... ). In order to reduce the uncertainty of the backscattered radar signal, it is necessary to average a certain number of independent samples. In the case of rain drops, since scatterers move with respect to each other, it is simpler to obtain a number of independent pulses that is larger than in the case of a terrain surface. The Swiss radars transmit 33 pulses within the HPBW at the lowest angle of elevation: in the case of ground clutter, only two or three pulses contribute to the reduction of the variance. The resulting probability distribution function (pdf) can in fact be better fitted by a lognormal or Weibull distribution. However, if a strong point target (such as the metallic tower on Cimetta) is present in the background of a radar cell that contains randomly distributed scatterers, the power distribution can be modeled using the Rice pdf [26]; in this case, the Log-transformed ratio between the (linear) average and the median power tends to be close to $0 \mathrm{~dB}$. Hence, Section 3.3.1 is focused on the average and median reflectivity values (complemented by the geometric average), while Section 3.3.2 describes the relative dispersion and the Log-distance between the $90 \%$ tile and the average.

\subsubsection{Central Location of the Radar Reflectivity: Three Location Parameters}

Three location parameters that describe the central location of the distribution of the radar reflectivity over the selected five days are shown in Tables 2 and 3 for horizontal and vertical polarization, respectively. The 2nd column shows the Log of the mean value, while the 3rd column shows the mean value of the log-transformed values: Since the algebraic average is always greater than (or equal to) the geometric average, it is obvious that the Log of the mean value is larger than 
the mean of the Log. However, it should be noted that the two central location values only differ by a fraction of $\mathrm{dB}$; this, in turn, implies the presence of a point target with a large radar cross section ("bright" scatterer). The last column shows the log-transformed median. It is easy to derive the Log-transformed ratio between the average and median reflectivity: It is simply the difference between the values in column 2 and those in column 4 . The five values of the horizontal polarization range from $0.03 \mathrm{~dB}$ (10 February 2015) to $0.39 \mathrm{~dB}$ (5 January 2015), which is typical of a Rice distribution that has a much larger contribution from the point target than from the background. The five values of the vertical polarization range from $-1.15 \mathrm{~dB}$ (10 February 2015) to $0.47 \mathrm{~dB}$ (18 January 2018), and are not compatible with a Rice distribution; they instead indicate a probability distribution function that is skewed to the left.

Table 2. Values of three central location parameters for the horizontal reflectivity distribution.

\begin{tabular}{cccc}
\hline Date & $\mathbf{1 0} \log (\overline{\mathbf{Z}})$ & $\overline{\mathbf{1 0} \log (\mathbf{Z})}$ & $\mathbf{1 0} \mathbf{L o g}\left(\boldsymbol{Z}_{\mathbf{5 0}}\right)$ \\
\hline 5 January 2015 & $81.89 \mathrm{dBZ}$ & $81.77 \mathrm{dBZ}$ & $81.5 \mathrm{dBZ}$ \\
9 February 2015 & $81.65 \mathrm{dBZ}$ & $81.59 \mathrm{dBZ}$ & $81.5 \mathrm{dBZ}$ \\
10 February 2015 & $82.03 \mathrm{dBZ}$ & $81.97 \mathrm{dBZ}$ & $82.0 \mathrm{dBZ}$ \\
13 October 2016 & $81.21 \mathrm{dBZ}$ & $81.12 \mathrm{dBZ}$ & $81.0 \mathrm{dBZ}$ \\
18 January 2017 & $81.08 \mathrm{dBZ}$ & $81.01 \mathrm{dBZ}$ & $81.0 \mathrm{dBZ}$ \\
\hline 5 days above & $81.59 \mathrm{dBZ}$ & $81.49 \mathrm{dBZ}$ & $81.5 \mathrm{dBZ}$ \\
\hline
\end{tabular}

Table 3. Same as Table 2, but for the vertical polarization.

\begin{tabular}{lccc}
\hline & $\mathbf{1 0} \log (\overline{\mathbf{Z}})$ & $\overline{\mathbf{1 0} \log (\mathbf{Z})}$ & $\mathbf{1 0} \mathbf{\operatorname { L o g } ( \mathbf { Z } _ { \mathbf { 5 0 } } )}$ \\
\hline 5 January 2015 & $80.20 \mathrm{dBZ}$ & $79.95 \mathrm{dBZ}$ & $80.0 \mathrm{dBZ}$ \\
9 February 2015 & $80.81 \mathrm{dBZ}$ & $80.73 \mathrm{dBZ}$ & $81.0 \mathrm{dBZ}$ \\
10 February 2015 & $80.85 \mathrm{dBZ}$ & $80.75 \mathrm{dBZ}$ & $82.0 \mathrm{dBZ}$ \\
13 October 2016 & $80.24 \mathrm{dBZ}$ & $80.08 \mathrm{dBZ}$ & $80.5 \mathrm{dBZ}$ \\
18 January 2017 & $79.97 \mathrm{dBZ}$ & $79.83 \mathrm{dBZ}$ & $79.5 \mathrm{dBZ}$ \\
\hline 5 days above & $80.43 \mathrm{dBZ}$ & $80.27 \mathrm{dBZ}$ & $80.5 \mathrm{dBZ}$ \\
\hline
\end{tabular}

3.3.2. Small Dispersion of the Reflectivity: Scale Parameters Based on Logarithmic and Linear Scales

Table 4 shows the two scale parameters that characterize the dispersion of the reflectivity distributions at the horizontal and vertical polarizations.

Table 4. Dispersion of the radar reflectivity values $(Z)$ for Horizontal $(H)$ and vertical $(V)$ polarization: Log-transformed ratio between the 90 percentile and the average (columns 2 and 3); ratio between the standard deviation and the average linear value of $\mathrm{Z}$ (columns 4 and 5).

\begin{tabular}{|c|c|c|c|c|}
\hline & $10 \cdot \log \left(Z H_{90} / \overline{Z H}\right)$ & $10 \cdot \log \left(Z V_{90} / \overline{Z V}\right)$ & $\operatorname{CoV}\{Z H\}$ & $\operatorname{CoV}\{Z V\}$ \\
\hline 5 January 2015 & $1.1 \mathrm{~dB}$ & $1.8 \mathrm{~dB}$ & 0.24 & 0.33 \\
\hline 9 February 2015 & $0.9 \mathrm{~dB}$ & $0.7 \mathrm{~dB}$ & 0.13 & 0.18 \\
\hline 10 February 2015 & $0.5 \mathrm{~dB}$ & $1.2 \mathrm{~dB}$ & 0.15 & 0.21 \\
\hline 13 October 2016 & $1.3 \mathrm{~dB}$ & $1.3 \mathrm{~dB}$ & 0.20 & 0.25 \\
\hline 18 January 2017 & $0.9 \mathrm{~dB}$ & $0.5 \mathrm{~dB}$ & 0.18 & 0.27 \\
\hline 5 days above & $0.91 \mathrm{~dB}$ & $1.07 \mathrm{~dB}$ & 0.20 & 0.26 \\
\hline
\end{tabular}

It is once again interesting to observe the Log-transformed ratio between the 90 percentile and the average because of the particular features of the Cimetta radar bin, with respect to other ground clutter bins. This Log-transformed ratio is found to be between 3 and $4 \mathrm{~dB}$ for a very large number of ground clutter bins; it is considerably smaller for the Cimetta bin: The most likely value is $0.9 \mathrm{~dB}$ for the horizontal polarization and $1.2 \mathrm{~dB}$ for the vertical polarization. 
Another parameter shown in Table 4 is the ratio between the standard deviation and the average linear value of $\mathrm{Z}$; this dimensionless quantity is often called the Coefficient of Variation (CoV) or relative dispersion. It should be noted that this value is exactly 1 for the Rayleigh backscatter model; it is significantly smaller than 1 for the Cimetta bin, which is affected to a great extent by the presence of the tall tower: the minimum (maximum) value was found on 9 February (5 January) 2015 for both polarizations. It is interesting to note that $\mathrm{CoV}\{Z V\}$ is larger than $\mathrm{CoV}\{Z H\}$ for all five days.

\subsection{On the Quite Stable Characteristics of the Differential Reflectivity}

In the absence of differential attenuation (which is the case of the five selected clear sky days), the differential reflectivity depends on the equivalent horizontal/vertical radar cross sections of the tower (its dielectric constant being the same for both orthogonal polarizations). In view of the results presented in Section 3.3, a positive $Z^{d r}$ of approximately $1 \mathrm{~dB}$ can be expected (by looking at the last column in Tables 2 and 3 , and it can be seen that the median of the 5 daily median reflectivity values is $81.5 \mathrm{dBZ}$ for the horizontal polarization and $80.5 \mathrm{dBZ}$ for the vertical polarization).

The last (central) column inf Table 5 shows the 5-daily median (average) differential reflectivity values (rounded to $0.1 \mathrm{~dB}$ ): in both cases, the median is $1.1 \mathrm{~dB}$, while the most likely values are $1.1 \mathrm{~dB}$ and $1.0 \mathrm{~dB}$ (4 cases out of 7 ). We can conclude that, on a daily basis, a positive median/average differential reflectivity of approximately $1 \mathrm{~dB}$ can be expected for the Cimetta tower. The first column in Table 5 shows another indicator of dispersion that we call "spread": it is half the distance between the 84 and the 16 percentiles, and it equals the standard deviation of a normal distribution. The (daily) standard deviation of the differential reflectivity is shown in the first column in Table 6; a comparison of these two columns shows that, using a $0.1 \mathrm{~dB}$ resolution, the spread of $Z^{d r}$ is undistinguishable from $\sigma\left\{Z^{d r}\right\}$ in 2 cases out of 5 ; in the other 3 cases, the spread is $0.1 \mathrm{~dB}$ larger than the standard deviation. Finally, the last two columns in Table 6 show the standard deviation of the Log-transformed radar reflectivity values of the horizontal and vertical polarizations. These values confirm what was shown in Section 3.3.2: the dispersion is larger for the vertical polarization than for the horizontal one for all of the five days.

Table 5. Spread, average and median values derived using 5-min differential reflectivity samples.

\begin{tabular}{cccc}
\hline & $\pm\left(\mathbf{Z}_{\mathbf{8 4}}^{d r}-\mathbf{Z}_{\mathbf{1 6}}^{\text {dr }}\right) / \mathbf{2}$ & $\overline{Z^{d r}}$ & $\mathbf{Z}_{\mathbf{5 0}}^{d r}$ \\
\hline 5 January 2015 & $\pm 0.7 \mathrm{~dB}$ & $1.8 \mathrm{~dB}$ & $1.7 \mathrm{~dB}$ \\
9 February 2015 & $\pm 0.7 \mathrm{~dB}$ & $0.8 \mathrm{~dB}$ & $0.7 \mathrm{~dB}$ \\
10 February 2015 & $\pm 0.9 \mathrm{~dB}$ & $1.1 \mathrm{~dB}$ & $1.1 \mathrm{~dB}$ \\
13 October 2016 & $\pm 1.0 \mathrm{~dB}$ & $1.0 \mathrm{~dB}$ & $1.0 \mathrm{~dB}$ \\
18 January 2017 & $\pm 1.1 \mathrm{~dB}$ & $1.1 \mathrm{~dB}$ & $1.1 \mathrm{~dB}$ \\
\hline
\end{tabular}

Table 6. Standard deviation of the differential reflectivity, the horizontal reflectivity and the vertical reflectivity.

\begin{tabular}{cccc}
\hline & $\pm \sigma\{\mathbf{1 0} \cdot \boldsymbol{\operatorname { l o g }}(\mathbf{Z H} / \mathbf{Z V})\}$ & $\pm \sigma\{\mathbf{1 0} \cdot \log (\mathbf{Z H})\}$ & $\pm \sigma\{\mathbf{1 0} \cdot \log (\mathbf{Z V})\}$ \\
\hline 5 January 2015 & $\pm 0.7 \mathrm{~dB}$ & $\pm 1.0 \mathrm{~dB}$ & $\pm 1.5 \mathrm{~dB}$ \\
9 February 2015 & $\pm 0.7 \mathrm{~dB}$ & $\pm 0.6 \mathrm{~dB}$ & $\pm 0.8 \mathrm{~dB}$ \\
10 February 2015 & $\pm 0.8 \mathrm{~dB}$ & $\pm 0.7 \mathrm{~dB}$ & $\pm 1.0 \mathrm{~dB}$ \\
13 October 2016 & $\pm 0.9 \mathrm{~dB}$ & $\pm 0.9 \mathrm{~dB}$ & $\pm 1.2 \mathrm{~dB}$ \\
18 January 2017 & $\pm 1.0 \mathrm{~dB}$ & $\pm 0.8 \mathrm{~dB}$ & $\pm 1.1 \mathrm{~dB}$ \\
\hline
\end{tabular}

\subsection{On the Remarkably Large and Stable Characteristics of the Copolar Correlation Coefficient}

The value range for $\rho^{H V}$ is between 0 (no correlation between the two polarizations) and 1 (perfect correlation). On the one hand, if the targets within the radar sampling volume were identical, then the time series of the signals at the horizontal and vertical polarizations would be perfectly correlated. 
On the other hand, the greater the variability in the shapes of the targets, the less likely it is that the two signals will be correlated. Hence, the copolar correlation coefficient is a good measurement of shape diversity: the light rain and drizzle and echoes (homogeneous liquid precipitation) are associated with very large values of $\rho^{H V}$, most of which are larger than 0.995 ; the $\rho^{H V}$ values in melting snow are lower (generally between 0.8 and 0.9 ) and make the melting layer easily distinguishable. If the sampling volume contains a significant number of large non-meteorological targets, such as biological targets (birds, insects etc.) or ground clutter, the $\rho^{H V}$ values will decrease considerably. The range of most ground clutter or anomalously propagated echoes is between 0.65 and 0.95 .

\subsubsection{Central Location of the Copolar Correlation Coefficient: Location Parameters}

As it can be seen from Table 7, the copolar correlation coefficient values for the Cimetta tower are remarkably large: the median (average) value of the 1440 samples (5-min temporal resolution over 5 days) is 0.9972 (0.9962). These values are typical of small spherical drops. As expected, the probability distribution functions are skewed to the left and the average value is smaller than the median for all of the five selected days.

Table 7. Values of five location parameters for the copolar correlation coefficient.

\begin{tabular}{cccccc}
\hline & Minimum & Average & Median & $\mathbf{9 0 \% \text { tile }}$ & MAX. \\
\hline 5 January 2015 & 0.9870 & 0.9944 & 0.9950 & 0.9971 & 0.9984 \\
9 February 2015 & 0.9925 & 0.9984 & 0.9985 & 0.9994 & 0.9998 \\
10 February 2015 & 0.9939 & 0.9976 & 0.9978 & 0.9994 & 0.9995 \\
13 October 2016 & 0.9882 & 0.9939 & 0.9941 & 0.9957 & 0.9976 \\
18 January 2017 & 0.9890 & 0.9966 & 0.9972 & 0.9983 & 0.9994 \\
\hline
\end{tabular}

\subsubsection{Small Dispersion of the Copolar Correlation Coefficient}

As it can be seen in Table 8, the dispersion around the mean of the $\rho^{H V}$ values is small: The standard deviation (multiplied by 100) of $\rho^{H V}$ for the five clear sky days ranges from between 0.09 and 0.23 .

Table 8. Values of spread and standard deviation for the copolar correlation coefficient.

\begin{tabular}{ccc}
\hline & $\left(\rho_{\mathbf{8 4}}^{H V}-\rho_{\mathbf{1 6}}^{H V}\right) / \mathbf{2}$ & $\left.\pm\left\{\sigma \rho^{H V}\right\}\right\}$ \\
\hline 5 January 2015 & 0.0025 & 0.0023 \\
9 February 2015 & 0.0007 & 0.0009 \\
10 February 2015 & 0.0008 & 0.0009 \\
13 October 2016 & 0.0012 & 0.0014 \\
18 January 2017 & 0.0017 & 0.0019 \\
\hline 5 days above & 0.0024 & 0.0024 \\
\hline
\end{tabular}

\subsection{On the Quite Stable Characteristics of the Differential Phase Shift}

The dispersion of the differential phase shift is relatively small over the 5 selected clear sky days: the standard deviation is $\sim 4^{\circ}$ in 4 days (out of 5), while it is $\sim 8^{\circ}$ on 18 January 2017. In turn, the standard deviation is similar to half the distance between the $84 \%$ tile and the $16 \%$ tile.

\section{Discussion}

From the values of the statistical parameters that characterize the radar observables presented in Section 3, it is easy to conclude that, on a daily basis, the BS signals acquired over the five selected clear sky days are statistically similar. This is why they were merged to derive a more representative 5-day data set (see the last line in Tables 2-4 and 8). Our strategy is that of trying to set up preliminary thresholds for warnings. The focus is on five (out of six) radar observables presented in Section 2.2, 
because a warning based on the differential phase shift of an individual radar bin is considered too risky.

As far as the Doppler spectrum width is concerned, since all 1440 values were $\mathrm{DN}=1$, a different value from $\mathrm{DN}=1$ would cause a warning. The reference framework for the Doppler velocity (1st Doppler moment) consists of 578 values with $\mathrm{DN}=128$ (zero velocity) and of 862 values with $\mathrm{DN}=127$. A different value would cause a warning.

As far as radar reflectivity is concerned, the median value results to be 81.5 (80.5) dBZ for the horizontal (vertical) polarization. The horizontal polarization shows a smaller standard deviation $( \pm 0.90 \mathrm{~dB})$ than the vertical one $( \pm 1.21 \mathrm{~dB})$. Hence, a rough monitoring of both polarizations is feasible. As a conservative rule of thumb, it can be decided that if the horizontal and vertical daily median reflectivity values were outside the [79.5-83.5] or [77.5-82.5] dBZ intervals (a little more than $\pm 2 \sigma$ ), a warning would be raised. Regarding the relative dispersion of linear $\mathrm{Z}$ a threshold of 0.30 (0.35) for the horizontal (vertical) polarization could be selected (see Table 4).

As far as the differential reflectivity is concerned, the 5-day mean and median values are $1.20 \mathrm{~dB}$ and $1.18 \mathrm{~dB}$, respectively. The standard deviation and spread are 0.89 and $0.99 \mathrm{~dB}$, respectively. Such dispersion is too large for monitoring purposes (see Section 2.4.4 and [19])

As far as the copolar correlation coefficient is concerned, the 5-day median (mean) value is 0.9968 (0.9962); the standard deviation and spread are both 0.0024 (last row of Table 8). Hence, the daily median of the copolar correlation coefficient could be expected to be larger than 0.994. Anyhow, if the median were smaller than 0.990 (conservative threshold) a warning would be raised.

We have tested the above-defined empirical intervals, using, as an example, data from another eleven clear sky days. In all cases, the spectrum width was always the same $(\mathrm{DN}=1)$, while the average radial velocity DN equaled 128 or 127 in all the cases but one (out of 34565 -min samples). Four warnings occurred on 28 September 2016. These were related to the following observables: Radial velocity ( $\mathrm{DN}=129$ once) and the coefficient of variation of both the horizontal $(\mathrm{CoV}=0.31)$ and vertical $(\mathrm{CoV}=0.48)$ reflectivity polarizations. On 22 February 2017, the $\mathrm{CoV}$ for the vertical polarization was as large as 0.50 (the standard deviation of $Z^{d r}$ was as large as $1.9 \mathrm{~dB}$ ). Hence, something anomalous had affected the vertical polarization. No anomalies were present for the following nine clear days: 6-7 January, 11 February 2015; 29 September, 3 and 15 October 2016; 21 April, 9 October 2017 and 22 November 2017 (note that on 21 April 2017, the standard deviation of $Z^{d r}$ was as large as $1.6 \mathrm{~dB}$ ). To summarize, nine (out of eleven) clear sky days shows good reproducibility of what had been statistically learnt from the training data set of five clear sky days.

Future research directions include the analysis of days characterized by heavy rain and/or very strong winds; these events should obviously be accompanied by an investigation regarding the stability and reproducibility of the Doppler and polarimetric signatures of the BS at a sub-daily temporal scale. However, it may be pointed out, just as an example, that during the last three hours of heavy rain (37 samples) that occurred on 16 June 2016, the median values of the horizontal and vertical reflectivity were as low as 77.5 and $75.0 \mathrm{dBZ}$ (two alerts). A third alert was associated to the median of $\rho^{H V}$, which was as low as 0.9872 . The standard deviation (36 degrees of freedom) of $Z^{d r}$ was also anomalously large $(1.5 \mathrm{~dB})$. It has been concluded that BS signals are remarkably weaker and less correlated during heavy rain.

\section{Conclusions and Outlook}

Thanks to the exponential increase in computational power for high resolution, intensive data processing and statistical analysis, a novel point of view regarding ground clutter has emerged in recent years: Its spatio-temporal characteristics are being investigated with the aim of monitoring the quality (and stability) of radar hardware. However, since most of ground distributed targets consist of a large number of randomly distributed scatterers, the corresponding backscattered signal is characterized by an extreme spatio-temporal variability in magnitude. So far, monitoring techniques have been based on the statistical parameters of the location (e.g., 90 percentile) of the reflectivity of a 
large number of radar bins that are above a given high reflectivity threshold (e.g., $55 \mathrm{dBZ}$ ) for more than a given percentage of time (e.g., $95 \%$ ).

In this note, we present an analysis that deals with the spectral (1st and 2nd Doppler moments) and polarimetric (differential reflectivity, copolar correlation coefficient and differential phase shift) information that characterizes an individual radar bin ("Bright Scatterer") which contains a dominant point target with deterministic backscattering properties in a background comprised of a random distribution of scatterers. The power backscattered by this BS is very large: It is around $80 \mathrm{dBZ}$ (at $18 \mathrm{~km}$ range), which corresponds to a power of approximately $-19 \mathrm{dBm}$, as received by the Lema radar. The particular Doppler and polarimetric signatures of the BS clearly emerge, so that, thanks to the statistical analysis presented in Section 3 of this note, empirical confidence intervals can be established for monitoring the reproducibility of weather radar observables (see Section 4). On the one hand, the check was successful in most of the analyzed clear sky days. On the other hand, the analysis of a heavy rain day shows 3 warnings: it can be argued that the backscattering properties of the BS in heavy rain conditions are remarkably different from those obtained for clear skies. Another field of future investigation is the study of the second-order properties of the various time series $(\mathrm{ZH}, \mathrm{ZV}$, $Z^{d r}, \rho^{H V}$, considered as random processes), merging several clear sky days; the study will be based on spectral analysis in order to reveal the diurnal (or shorter) components, scaling behavior over temporal scales and their auto-correlation (or power spectrum).

Acknowledgments: The author would like to thank Daniel Wolfensberger, Floortje van den Heuvel, Alexis Berne, Urs Germann, Maurizio Sartori and Marco Boscacci for the stimulating discussions. He would also like to thank the anonymous reviewers for their helpful and valuable suggestions.

Conflicts of Interest: The author declares no conflict of interest.

\section{References}

1. Wallace, P.R. Interpretation of the fluctuating echoes from randomly distributed scatterers, part II. Can. J. Phys. 1953, 31, 995-1009. [CrossRef]

2. Linell, T. An Experimental Investigation of the Amplitude Distribution of Radar Terrain Return; Institute of National Defense: Taoyuan City, Taiwan, 1966.

3. Boothe, R.R. The Weibull Distribution Applied to the Ground Clutter Backscatter Coefficient; Tech. Rep. No. RE-69-15; U.S. Army Missile Command: Redstone Arsenal, AL, USA, 1969.

4. Hubbert, J.C.; Dixon, M.; Ellis, S.M.; Meymaris, G. Weather Radar Ground-clutter. Part I: Identification, Modeling, and Simulation. J. Atmos. Ocean. Technol. 2009, 26, 1165-1185. [CrossRef]

5. Silberstein, D.S.; Wolff, D.B.; Marks, D.A.; Atlas, D.; Pippitt, J.L. Ground Clutter as a Monitor of Radar Stability at Kwajalein, RMI. J. Atmos. Ocean. Technol. 2008, 25, 2037-2045. [CrossRef]

6. Bertoldo, S.; Notarpietro, R.; Gabella, M.; Perona, G. Ground clutter analysis to monitor the stability of a mobile X-band radar. In Proceedings of the 9th International Workshop on Precipitation in Urban Areas, St. Moritz, Switzerland, 6-9 December 2012.

7. Wolff, D.B.; Marks, D.A.; Petersen, W.A. General Application of the Relative Calibration Adjustment (RCA) Technique for Monitoring and Correcting Radar Reflectivity Calibration J. Atmos. Ocean. Technol. 2015, 32, 496-506. [CrossRef]

8. Gabella, M.; Sartori, M.; Progin, O.; Boscacci, M.; Germann, U. An innovative instrumentation for checking electromagnetic performances of operational meteorological radar. In Proceedings of the 6th European Conference on Radar in Meteorology and Hydrology (ERAD2010), Sibiu, Romania, 6-10 September 2010; pp. 263-269.

9. Huuskonen, A.; Holleman, I. Determining weather radar antenna pointing using signals detected from the Sun at low antenna elevations. J. Atmos. Ocean. Technol. 2007, 24, 476-483. [CrossRef]

10. Holleman, I.; Huuskonen, A.; Kurri, M.; Beekhuis, H. Operational monitoring of weather radar receiving chain using the Sun. J. Atmos. Ocean. Technol. 2010, 27, 159-166. [CrossRef]

11. Gabella, M.; Sartori, M.; Boscacci, M.; Germann, U. Vertical and Horizontal Polarization Observations of Slowly Varying Solar Emissions from Operational Swiss Weather Radars. Atmosphere 2015, 6, 50-59. [CrossRef] 
12. Huuskonen, A.; Kurri, M.; Holleman, I. Improved analysis of Solar signals for differential reflectivity monitoring. Atmos. Meas. Technol. 2016, 9, 3183-3192. [CrossRef]

13. Gabella, M.; Boscacci, M.; Sartori, M.; Germann, U. Calibration accuracy of the dual-polarization receivers of the C-band Swiss weather radar network. Atmosphere 2016, 7, 76. [CrossRef]

14. Reinhart, R.E. On the use of ground return targets for radar reflectivity factor calibration checks. J. Appl. Meteorol. 1978, 17, 1342-1350. [CrossRef]

15. Melnikov, V.M.; Zrnic, D.S.; Doviak, R.J.; Carter, J.K. Calibration and Performance Analysis of NSSL'S Polarimetric WSR-88D; National Sever Storms Laboratory: Norman, Oklahoma, 2003. Available online: https://www.nssl.noaa.gov/publications/wsr88d_reports/Calibration_and_Performance_Analysis.pdf (accessed on 29 May 2018).

16. Germann, U.; Boscacci, M.; Gabella, M.; Sartori, M. Radar design for prediction in the Swiss Alps. Meteorol. Technol. Int. 2015, 4, 42-45.

17. Vollbracht, D.; Sartori, M.; Gabella, M. Absolute dual-polarization radar calibration: Temperature dependence and stability with focus on antenna-mounted receivers and noise source-generated reference signal. In Proceedings of the 8th European Conference on Radar in Meteorology and Hydrology (ERAD2014), Garmisch-Partenkirchen, Germany, 1-5 September 2014.

18. Seliga, T.; Bringi, V. Potential use of radar differential reflectivity measurements at orthogonal polarizations for measuring precipitation. J. Appl. Meteorol. 1976, 15, 69-76. [CrossRef]

19. Illingworth, A. Improved precipitation rates and data quality by using polarimetric measurements. In Advanced Applications of Weather Radar; Meischner, P., Ed.; Springer: Berlin/Heidelberg, Germany, 2004; Chapter 5; pp. 130-166.

20. Pratte, J.F.; Ferraro, D.G. Automated solar gain calibration. In Proceedings of the 24th Conference on Radar Meteorology, Tallahassee, FL, USA, 27-31 March 1989; pp. 619-622.

21. Ryzhkov, A.V.; Giangrande, S.E.; Melnikov, V.M.; Schuur, T.J. Calibration issues of dual-polarization radar measurements. J. Atmos. Ocean. Technol. 2005, 22, 1138-1155. [CrossRef]

22. Gabella, M.; Huuskonen, A.; Sartori, M.; Holleman, I.; Boscacci, M.; Germann, U. Evaluating the Solar Slowly Varying Component at C-Band Using Dual- and Single-Polarization Weather Radars in Europe. Adv. Meteorol. 2017, 2017, 4971765. [CrossRef]

23. Hubbert, J.C. Differential Reflectivity Calibration and Antenna Temperature. J. Atmos. Ocean. Technol. 2017, 34, 1885-1906. [CrossRef]

24. Fabry, F. Radar Meteorology: Principles and Practice, 1st ed.; Cambridge University Press: Cambridge, UK, 2015; ISBN 978-1-107-07046-2.

25. Bringi, V.N.; Chandrasekar, V. Polarimetric Doppler Weather Radar: Principles and Applications, 1st ed.; Cambridge University Press: Cambridge, UK, 2001.

26. Beckmann, P. Probability in Communication Engeneering, 1st ed.; Harcourt, Brace, and World: New York, NY, USA, 1967.

(C) 2018 by the author. Licensee MDPI, Basel, Switzerland. This article is an open access article distributed under the terms and conditions of the Creative Commons Attribution (CC BY) license (http:// creativecommons.org/licenses/by/4.0/). 